\title{
Soliton solutions for Korteweg-de Vries equation by homotopy analysis method
}

\author{
H. Song ${ }^{1} \quad$ L. Tao $^{2}$
}

(Received 18 July 2008; revised 15 October 2008)

\begin{abstract}
This article solves the well-known Korteweg-de Vries equation by the homotopy analysis method, an analytical, totally explicit technique. By choosing a proper auxiliary parameter, the new series solution converges rapidly to the exact solution, with a simple way to adjust the convergence region. In addition, we show that a significant improvement of the convergence rate and region is achieved by applying homotopy-padé approximants. The present method holds promise in providing soliton solutions for more complicated wave equations.
\end{abstract}

\section{Contents}

\section{Introduction}

http://anziamj . austms.org.au/ojs/index.php/ANZIAMJ/article/view/1354 gives this article, (C) Austral. Mathematical Soc. 2008. Published October 29, 2008. ISSN 1446-8735. (Print two pages per sheet of paper.) 


\section{Theoretical consideration}

2.1 The basic idea behind HAM . . . . . . . . . . . . . C154

2.2 Soliton solution of KdV equation by HAM . . . . . . . C C156

3 Result and discussion

4 Conclusion

\section{Introduction}

Most physical problem in the real world are nonlinear and in turn are described by nonlinear equations. However, it is generally difficult to solve nonlinear differential equations accurately by analytical means. Therefore, seeking suitable solving methods is an active task in branches of computational physics. Recently, a new analytic approach named homotopy analysis method (HAM) has seen rapid development. It has been successfully applied to many nonlinear problems and logically contains Lyapunov's small parameter method, the $\delta$-expansion method, and Adomian's decomposition method [4]. Without depending on a small parameter such as in a perturbation approach, the HAM has a particular advantage in solving strong nonlinear problems. Other advantages associated with the HAM over the perturbation technique include greater flexibility in the selection of a proper set of base functions for the solution and a much simpler way in the control of the convergence rate and region. The HAM was first applied to fluid mechanics by Liao $[3,4]$. Liao and Cheung [5] successfully applied HAM in fully analytical way to nonlinear waves propagating in deep water and the HAM solution in finite water depth was later obtained by Tao et al. [7]. Abbasbandy [1], Wu and Liao [8], and Rashidi et al. [9] also obtained HAM solutions for some shallow water wave equations. 
Among many nonlinear equations in physics, The Korteweg-de Vries $(\mathrm{KdV})$ equation is a typical, relatively simple and classical one. It is a wellknown mathematical model of nonlinear waves on shallow water surfaces. The so-called "soliton" originated from solving the Kdv equation. Many researchers still endeavour to solve KdV-type equations [2, 6, e.g.]. In this article, the HAM is applied to obtain soliton solutions for the KdV equation. An explicit solution is presented and compared with the exact solution. Very good agreement is achieved, demonstrating the high efficiency of HAM.

\section{Theoretical consideration}

\subsection{The basic idea behind HAM}

Consider a nonlinear equation

$$
\mathcal{N}[\mathrm{f}(\mathrm{x}, \mathrm{t})]=0,
$$

where $\mathcal{N}$ is a nonlinear operator, $f(x, t)$ is the function to be found, and $\mathbf{x}$ and $\mathbf{t}$ are spatial and temporal independent variables respectively.

HAM is based on a continuous variation from an initial trial to the exact solution. By constructing the homotopic mapping $f(x, t) \rightarrow F(x, t ; q)$, we have the following homotopy:

$$
(1-q) \mathcal{L}\left[F(x, t ; q)-f_{0}(x, t)\right]=q \hbar H(x, t) \mathcal{N}[F(x, t ; q)], \quad q \in[0,1],
$$

where $F(x, t ; q)$ is differentiable with respect to the embedding parameter $q$, $f_{0}(x, t)$ is an initial estimate of $f(x, t), \hbar$ is a nonzero auxiliary parameter, $\mathrm{H}(\mathrm{x}, \mathrm{t})$ is a nonzero auxiliary function, and $\mathcal{L}$ is a linear auxiliary operator with the property of $\mathcal{L}[0]=0$.

When $\mathrm{q}=0$ and $\mathrm{q}=1$, it holds

$$
\mathrm{F}(\mathrm{x}, \mathrm{t} ; 0)=\mathrm{f}_{\mathrm{o}}(\mathrm{x}, \mathrm{t}), \quad \mathcal{N}[\mathrm{F}(\mathrm{x}, \mathrm{t} ; 1)]=0,
$$


respectively. Therefore, as the embedding parameter $\mathbf{q}$ varies from 0 to 1 , $F(x, t ; q)$ maps continuously from the initial estimate $f_{0}(x, t)$ to the desired solution $f(x, t)$.

By Taylor's theorem, $\mathrm{F}(\mathbf{x}, \mathbf{t} ; \mathbf{q})$ is expanded with respect to the embedding parameter $\mathbf{q}$ as

$$
F(x, t ; q)=f_{o}(x, t)+\sum_{m=1}^{\infty} f_{m}(x, t) q^{m}
$$

where

$$
f_{m}(x, t)=\left.\frac{1}{m !} \frac{\partial^{m} F(x, t ; q)}{\partial q^{m}}\right|_{q=0} .
$$

Differentiating the zeroth order deformation equation (2) $m$ times with respect to $\mathrm{q}$ at $\mathrm{q}=0$ and then dividing it by $\mathrm{m}$ !, we have the following mth order deformation equation

$$
\mathcal{L}\left[f_{m}(x, t)-\chi_{m} f_{m-1}(x, t)\right]=\hbar R_{m}(x, t),
$$

where

$$
\begin{aligned}
& x_{m}= \begin{cases}0, & m \leq 1 \\
1, & m>1,\end{cases} \\
& R_{m}(x, t)=\left.\frac{1}{(m-1) !} \frac{\partial^{m-1} \mathcal{N}[F(x, t ; q)]}{\partial q^{m-1}}\right|_{q=0} .
\end{aligned}
$$

If the series (4) converges at $\mathbf{q}=1$, then the desired solution

$$
f(x, t)=f_{0}(x, t)+\sum_{m=1}^{\infty} f_{m}(x, t) .
$$

Given a nonlinear problem, we choose a set of base functions to present its solutions, which provides us with the rule of solution expression [4]. The 
auxiliary parameter $\hbar$ provides a convenient way to control and adjust the rate and region of the convergence [4]. The dependence of HAM solution on $\hbar$ and detailed discussions are presented in Section 3. The auxiliary function $\mathrm{H}(\mathbf{x}, \mathbf{t})$ in the zeroth order deformation equation (2) is determined by the so called rule of coefficient ergodicity [4], that is, each base should appear in the solution expression at least once. However, in most cases including in this article, $\mathbf{H}(\mathbf{x}, \mathbf{t})$ is chosen as 1 and vanishes from equation (2).

\subsection{Soliton solution of $\mathrm{KdV}$ equation by $\mathrm{HAM}$}

The Kdv equation is the nonlinear, dispersive partial differential equation

$$
u_{t}+u_{x x x}+6 u u_{x}=0
$$

where $x$ is space, $t$ is time and subscripts designate partial derivation with respect to the variable.

For travelling wave solutions, defining $\theta=x-$ ct and $u(\theta)=a v(\theta)$, equation (10) is

$$
v^{\prime \prime \prime}(\theta)+6 a v(\theta) v^{\prime}(\theta)-c v^{\prime}(\theta)=0,
$$

where $\mathrm{c}$ is the wave speed, $\boldsymbol{a}$ is the wave amplitude, and a prime designates the derivative with respect to $\theta$.

For a one-loop soliton solution, it is natural to locate the origin at the position where the nondimensional wave elevation $v(\theta)$ achieves its maximum. We then have the boundary conditions at the origin and infinity as

$$
v(0)=1, \quad v^{\prime}(0)=0, \quad v(+\infty)=0 .
$$

As the soliton wave evanesces at infinity, we assume

$$
v(\theta) \sim \mathrm{B} \exp (-\lambda \theta) \text { as } \theta \rightarrow+\infty,
$$


where $\lambda>0$ and B are constants. Substituting equation (13) into equation (11) and balancing the main terms, we have

$$
\lambda=\sqrt{c} .
$$

Define $\tau=\lambda \theta$, equation (11) becomes

$$
c v^{\prime \prime \prime}(\tau)+6 a v(\tau) v^{\prime}(\tau)-c v^{\prime}(\tau)=0
$$

with the boundary conditions (12).

Then the solution is expressed in terms of the base functions

$$
\{\exp (-n \tau) \mid n=1,2,3, \ldots\},
$$

in the form

$$
v(\tau)=\sum_{n=1}^{+\infty} b_{n} \exp (-n \tau),
$$

where $b_{n}$ is a coefficient to be determined. The nonlinear operator $\mathcal{N}$ is chosen as

$$
\mathcal{N}[\Phi(\tau ; \mathbf{q}), A(\mathbf{q})]=c \frac{\partial^{3} \Phi(\tau ; \mathbf{q})}{\partial \tau^{3}}+6 \mathrm{~A}(\mathbf{q}) \Phi(\tau ; \mathbf{q}) \frac{\partial \Phi(\tau ; \mathbf{q})}{\partial \tau}-c \frac{\partial \Phi(\tau ; \mathbf{q})}{\partial \tau}
$$

where $\Phi(\tau ; \mathbf{q})$ and $A(\mathbf{q})$ are the mapping functions of $v(\tau)$ and a respectively. The linear operator $\mathcal{L}$ is chosen as

$$
\mathcal{L}[\Phi(\tau ; \mathbf{q})]=\left(\frac{\partial^{3}}{\partial \tau^{3}}-\frac{\partial}{\partial \tau}\right) \Phi(\tau ; q)
$$

which has the property

$$
\mathcal{L}\left[\mathrm{C}_{1} \exp (-\tau)+\mathrm{C}_{2} \exp (\tau)+\mathrm{C}_{3}\right]=0,
$$

where $C_{1}, C_{2}$ and $C_{3}$ are constants. 
According to the boundary conditions (12) and the rule of solution expression (17), the initial guess is chosen as

$$
\phi_{0}(\tau)=2 \exp (-\tau)-\exp (-2 \tau)
$$

The zeroth order deformation equation is

$$
(1-q) \mathcal{L}\left[\Phi(\tau ; q)-\phi_{0}(\tau ; q)\right]=q \hbar \mathcal{N}[\Phi(\tau ; q), A(q)]
$$

subject to the boundary conditions

$$
\Phi(0 ; q)=1, \quad \Phi_{\tau}(0 ; q)=0, \quad \Phi(+\infty ; q)=0 .
$$

Expand $\Phi(0, \mathbf{q})$ and $A(\mathbf{q})$ in Taylor series with respect to $\mathbf{q}$, we have

$$
\begin{aligned}
& \Phi(\tau ; q)=\phi_{0}(\tau)+\sum_{m=1}^{\infty} \phi_{m}(\tau) q^{m} \\
& A(q)=a_{0}+\sum_{m=1}^{\infty} a_{m} q^{m}
\end{aligned}
$$

where

$$
\begin{aligned}
& \phi_{m}(\tau)=\left.\frac{1}{m !} \frac{\partial^{m} \Phi(\tau ; q)}{\partial q^{m}}\right|_{q=0} \\
& a_{m}=\left.\frac{1}{m !} \frac{\partial^{m} A(q)}{\partial q^{m}}\right|_{q=0}
\end{aligned}
$$

For brevity, we define

$$
\vec{\phi}_{m}=\left\{\phi_{0}, \phi_{1}, \phi_{2}, \ldots, \phi_{m}\right\} \quad \text { and } \quad \vec{a}_{m}=\left\{a_{0}, a_{1}, a_{2}, \ldots, a_{m}\right\} .
$$

Differentiating equations (22) and (23) $\mathrm{m}$ times with respect to $\mathbf{q}$ at $\mathbf{q}=0$, and then dividing them by $m$ !, the $m$ th order deformation equation is

$$
\mathcal{L}\left[\phi_{m}(\tau)-\chi_{m} \phi_{m-1}(\tau)\right]=\hbar R_{m}\left(\vec{\phi}_{m}, \vec{a}_{m}\right),
$$


subject to the boundary conditions

$$
\phi_{\mathfrak{m}}(0)=\phi_{\mathfrak{m}}(+\infty)=\phi_{\mathfrak{m}}^{\prime}(0)=0
$$

where

$$
\mathrm{R}_{\mathrm{m}}\left(\vec{\phi}_{\mathrm{m}-1}, \overrightarrow{\mathrm{a}}_{\mathrm{m}-1}\right)=\mathrm{c} \phi_{m-1}^{\prime \prime \prime}+6 \sum_{i=0}^{\mathrm{m}-1} \sum_{j=0}^{i} a_{j} \phi_{i-j} \phi_{m-i-1}^{\prime}-c \phi_{m-1}^{\prime} .
$$

The solution of equation (29) is

$$
\phi_{\mathfrak{m}}(\tau)=C_{1} \exp (-\tau)+C_{2} \exp (\tau)+C_{3}+\hat{\phi}_{m}(\tau),
$$

where $\hat{\phi}_{\mathfrak{m}}(\tau)$ is a particular solution of equation (29) with unknown term $a_{m-1}$. According to the boundary condition (30) and rule of solution expression (17),

$$
\mathrm{C}_{2}=\mathrm{C}_{3}=0, \quad \mathrm{C}_{1}=-\hat{\phi}_{\mathrm{m}}(0) \text { and } \hat{\phi}_{\mathrm{m}}(0)+\hat{\phi}_{\mathrm{m}}^{\prime}(0)=0,
$$

which determine $a_{m-1}$.

\section{Result and discussion}

The convergence region and rate are controlled by the auxiliary parameter $\hbar$ in HAM. For different value of $\hbar$, a converges to the same value - the approximation of the exact solution. As shown in Figure 1, the nearly horizontal line segments of $a-\hbar$ curves correspond to the convergence regions of the $\hbar$ values. Figure 1 clearly shows that the first $2 m$ th order approximations of the $a / c$ converge in a region around $\hbar \in[-2,-1 / 2]$. The convergence region enlarges as more high order terms are included in the series. Based on the above arguments, the auxiliary parameter is chosen as $\hbar=-1$ for all the HAM solutions presented in this section. 


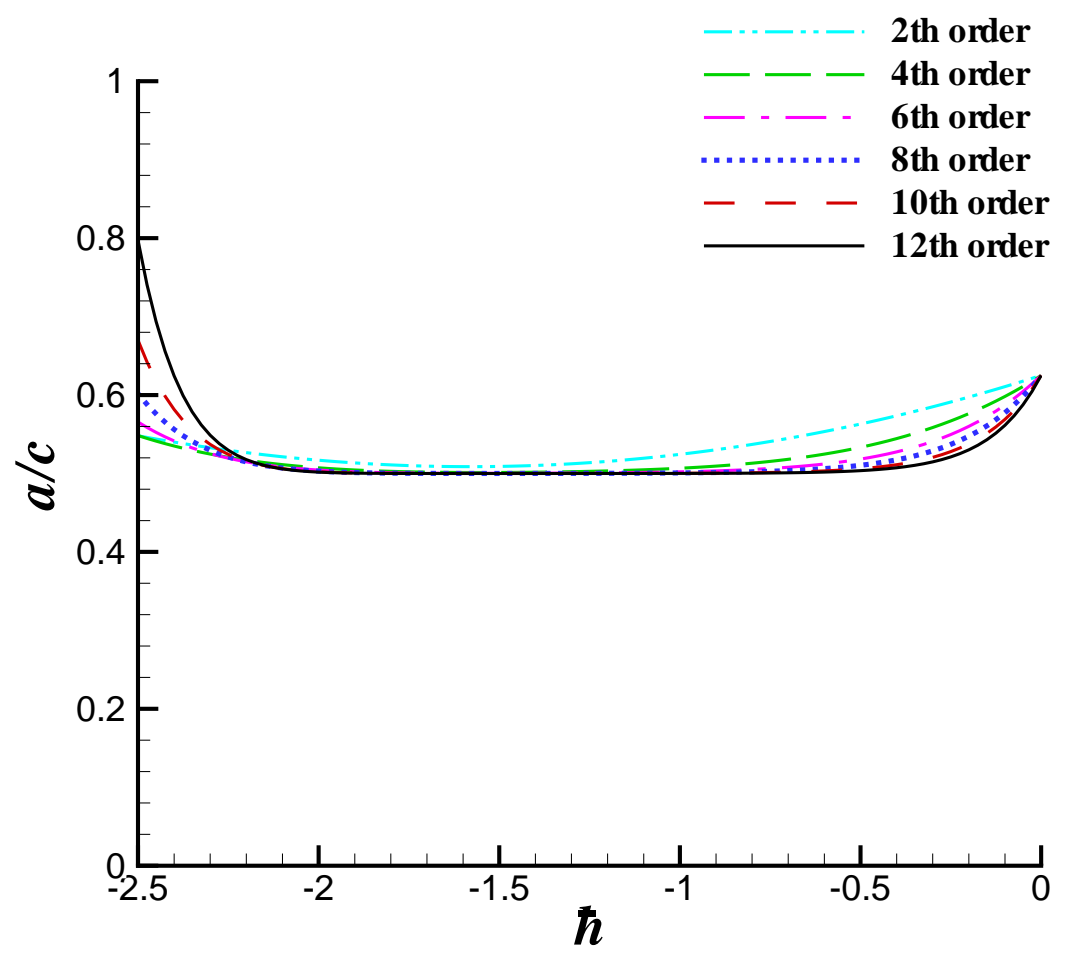

Figure 1: The first $2 m$ th order approximations of $a / c$ versus $\hbar$. 
Since the KdV equation is integrable, the exact soliton solution of (10) is

$$
u(x, t)=\frac{c}{2 \cosh ^{2}\left[\frac{\sqrt{c}}{2}(x-c t-d)\right]},
$$

where $\mathrm{d}$ is an arbitrary constant, chosen as 0 in the present coordinates.

The zeroth, first and second order approximations of $v(\tau)$ and $a$ are (recall $\mathfrak{u}(\theta)=\mathfrak{a} v(\theta), \tau=\sqrt{\mathrm{c}} \theta$ and $\theta=x-c t)$

$$
\begin{aligned}
\tilde{v}_{0}(\tau)= & 2 e^{-\tau}-e^{-2 \tau}, \\
\tilde{v}_{1}(\tau)= & \frac{43}{16} e^{-\tau}-\frac{5}{2} e^{-2 \tau}+\frac{15}{16} e^{-3 \tau}-\frac{1}{8} e^{-4 \tau}, \\
\tilde{v}_{2}(\tau)= & \frac{12905}{4096} e^{-\tau}-\frac{14065}{3584} e^{-2 \tau}+\frac{73275}{28672} e^{-3 \tau} \\
& -\frac{13677}{14336} e^{-4 \tau}+\frac{95}{512} e^{-5 \tau}-\frac{3}{224} e^{-6 \tau}, \\
\tilde{\mathrm{a}}_{0}= & \frac{5}{8} \mathrm{c}, \\
\tilde{\mathrm{a}}_{1}= & \frac{7905}{14336} \mathrm{c}, \\
\tilde{\mathrm{a}}_{2}= & \frac{20207405}{38535168} \mathrm{c},
\end{aligned}
$$

where the tilde designates the approximation solution and the number in the subscript denotes the order number.

Choosing the first three terms of series (24) and (25) respectively and using the built-in function PadeApproximant in Mathematica 6 , the $[1,1]$ homotopy-padé (HP) approximation of $\mathcal{v}(\tau)$ and $a$ are

$$
\begin{aligned}
\tilde{v}_{[1,1]}(\tau) & =\frac{4054+64 e^{-2 \tau}-504 e^{-\tau}-17069 e^{\tau}+26418 e^{2 \tau}}{384-4552 e^{\tau}+10698 e^{2 \tau}+6433 e^{3 \tau}}, \\
\tilde{a}_{[1,1]} & =\frac{2921395}{5742736} c .
\end{aligned}
$$


TABLE 1: The $2 m$ th order solution and $[m, m]$ homotopy-padé approximation of $\mathrm{a} / \mathrm{c}$ for $\hbar=-1$.

\begin{tabular}{cccccc}
\hline Order & $\mathrm{a} / \mathrm{c}$ & Error & {$[\mathrm{m}, \mathrm{m}]$} & $\mathrm{a} / \mathrm{c}$ & Error \\
\hline 2 & 0.524389 & $4.8778 \%$ & {$[1,1]$} & 0.508711 & $1.7422 \%$ \\
4 & 0.506689 & $1.3378 \%$ & {$[2,2]$} & 0.500734 & $0.1468 \%$ \\
6 & 0.502126 & $0.4252 \%$ & {$[3,3]$} & 0.500055 & $0.0110 \%$ \\
8 & 0.500737 & $0.1474 \%$ & {$[4,4]$} & 0.500005 & $0.0010 \%$ \\
10 & 0.500271 & $0.0542 \%$ & {$[5,5]$} & 0.500000 & $0.0000 \%$ \\
\hline Exact & & & $1 / 2$ & & \\
\hline
\end{tabular}

The comparison of the HAM solution and the exact solution is shown in Table 1 , where the first $2 \mathrm{~m}$ th order solution and $[\mathrm{m}, \mathrm{m}]$ homotopy-padé approximation of $\mathrm{a} / \mathrm{c}$ for $\hbar=-1$ are shown. A rapid convergence rate of the series is evident. Table 1 also shows that the present homotopypadé giving high accurate results with only a few number of terms (the relative error is $1.74 \%$ for $[1,1] \mathrm{HP}$ approximation and $0.15 \%$ for $[2,2] \mathrm{HP}$ approximation). This is a further demonstration of the excellent convergence rate in the present homotopy-padé technique.

The first $2 m$ th order solution and [ $m, m]$ homotopy-padé approximation of $u$ versus $\theta$ for $\hbar=-1$ and $c=1$ is shown in Figure 2. The present HAM solution is almost identical with the exact solution especially for homotopypadé solutions as shown in Figure 2. Even the $[1,1]$ HP approximation yields very good agreement. It is also evident that the higher order approximations result in better accuracy.

\section{Conclusion}

In this article, explicit solutions of the well-known Kdv equation were derived using the homotopy analysis method. Different from perturbation 


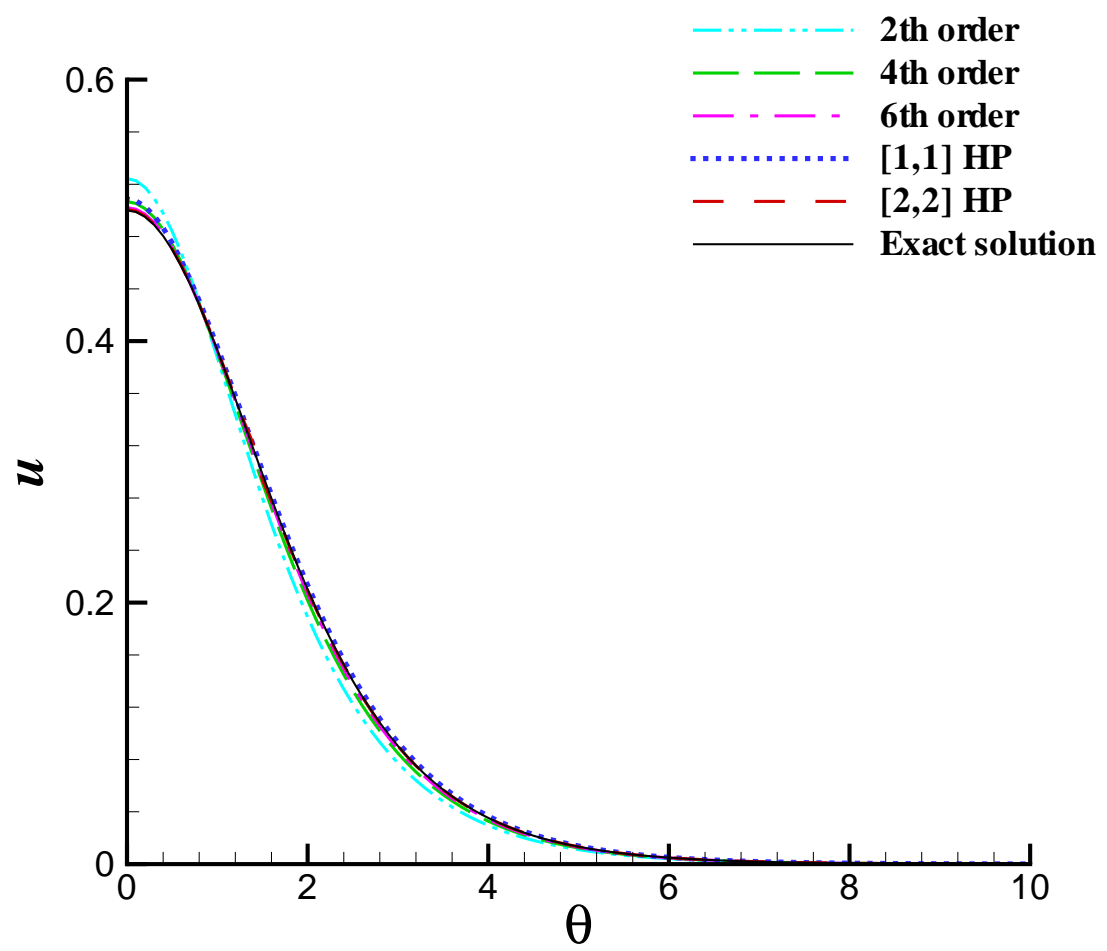

FiguRE 2: The first $2 m$ th order solution and $[m, m]$ homotopy-padé approximation of $u$ versus $\theta$ for $\hbar=-1$ and $c=1$. 
techniques, the present HAM approach is not dependent on any small or large parameter, and is particularly suitable for solving nonlinear problems. The convergence region is controlled by the non-zero parameter $\hbar$, providing us a simple way to adjust convergence. Furthermore, a significant improvement of the convergence rate and region is achieved by applying homotopy-padé technique. The present method holds promise in providing soliton solutions for more complicated wave equations.

Acknowledgements This article is based on the project funded by Australian Research Council under Discovery Project Grant DP0450906. The first author is grateful for a postdoctoral fellowship from Griffith University to support this research.

\section{References}

[1] Abbasbandy, S., Homotopy analysis method for generalized Benjamin-Bona-Mahony equation, Z. angew. Math. Phys., 59, 2008, 51-62. doi:10.1007/s00033-007-6115-x C153

[2] Fan, E., A new algebraic method for finding the line soliton solutions and doubly periodic wave solution to a two-dimensional perturbed KdV equation, Chaos Soliton Fract., 15, 2003, 567-574. doi:10.1016/S0960-0779(02)00146-7 C154

[3] Liao, S. J., An approximate solution technique not depending on small parameters: a special example, Int. J. Nonlinear Mech., 30, 1995, 371-380. doi:10.1016/0020-7462(94)00054-E C153

[4] Liao, S. J., Beyond Perturbation: Introduction to the Homotopy Analysis Method. Chapman \& Hall/CRC, Florida, 2004. C153, C155, C156 
[5] Liao, S. J. and Cheung, K. F., Homotopy analysis of nonlinear progressive waves in deep water, J. Eng. Math., 45, 2003, 105-116. doi:10.1023/A:1022189509293 C153

[6] Ping, H., Zheng, C. and Jun, F., Solution of Kdv equation by computer algebra, Appl. Math. Comput., 136, 2003, 511-515. doi:10.1016/S0096-3003(02)00076-0 C154

[7] Tao, L., Song, H. and Chakrabarti, S., Nonlinear progressive waves in water of finite depth-an analytic approximation, Coast. Eng., 54, 2007, 825-834. doi:10.1016/j.coastaleng.2007.05.008 C153

[8] Wu, W. and Liao, S. J., Solving solitary waves with discontinuity by means of the homotopy analysis method, Chaos Soliton Fract., 26, 2005, 177-185. doi:10.1016/j.chaos.2004.12.016 C153

[9] Rashidi, M. M., Ganji, D. D. and Dinarvand S., Approximate traveling wave solutions of coupled Whitham-Broer-Kaup shallow water equations by homotopy analysis method, Differential Equations and Nonlinear Mechanics, 2008, Article ID 243459, doi:10.1155/2008/243459 C153

\section{Author addresses}

1. H. Song, Griffith School of Engineering, Griffith University, Queensland 4222, Australia. mailto:h.song@griffith.edu.au

2. L. Tao, Griffith School of Engineering, Griffith University, Queensland 4222, Australia. 